\title{
Combining Two-Directional Synthesis and Tandem Reactions: Synthesis of Trioxadispiroketals
}

\author{
Paul J. McDermott and Robert A. Stockman* \\ School of Chemical Sciences and Pharmacy, University of East Anglia, Norwich,UK, \\ NR4 7TJ Fax+44(1603)592003, r.stockman@uea.ac.uk.
}

\section{Contents:}

- Experimental procedures and spectral data for compounds 6, 7and 8

- Experimental procedures and spectral data for compounds 11, 12, 13 and 14

- $\quad{ }^{1} \mathrm{H}-\mathrm{NMR}$ and ${ }^{13} \mathrm{C}-\mathrm{NMR}$ spectra for compounds $\mathbf{6 , 7 , 8 , 1 1 , 1 2 , 1 3}$ and 14 
3-[5(2-Methoxycarbonyl-vinyl-)-furan-2-yl]-acrylic acid methyl ester

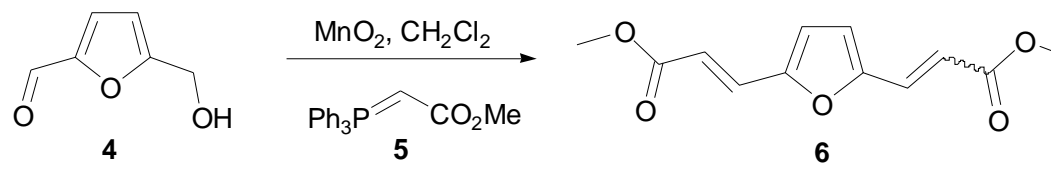

A mixture of 5-hydroxymethyl-2-furaldehyde $(1 \mathrm{~g}, \quad 7.9 \mathrm{mmol})$, (methoxycarbonylmethylen)-triphenylphosphorane 5 (6.36g, $19 \mathrm{mmol}, 2.4 \mathrm{eq}$ ) and manganese (iv) oxide $(6.89 \mathrm{~g}, 79 \mathrm{mmol}, 10 \mathrm{eq})$ in $\mathrm{CH}_{2} \mathrm{Cl}_{2}(20 \mathrm{~mL})$ was stirred under argon for 4 days. The reaction mixture was filtered through celite and reduced in vacuo. Purification by column chromatography over silica gel (eluting with 1:1 petrol ether/ethyl acetate) gave 3-[5-(2-Methoxycarbonyl-vinyl-)-furan-2-yl]-acrylic acid methyl ester $6(1.63 \mathrm{~g}, 87 \%)$ as a yellow crystalline solid.

\section{Spectral Data for the 2 isomers:}

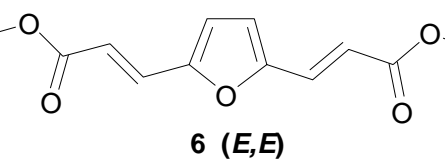

IR: 2923, 2853, 1727, 1640, 1461. MS: m/z = [M+H] 236.1 (100), 205.1 (95), 63.2 (98). HRMS: Calcd for $\mathrm{C}_{12} \mathrm{H}_{12} \mathrm{O}_{5}[\mathrm{M}+\mathrm{H}]:$ 237.0757. Found: 237.0757. $\delta_{\mathrm{H}}(400 \mathrm{MHz}$, $\left.\mathrm{CDCl}_{3}\right) ; 7.33\left(2 \mathrm{H}, \mathrm{d}, \mathrm{J}\right.$ 15.7), $6.56(2 \mathrm{H}, \mathrm{s}), 6.36(2 \mathrm{H}, \mathrm{d}, \mathrm{J} 15.7), 3.74(3 \mathrm{H}, \mathrm{s}) . \delta_{\mathrm{C}}(400$ $\left.\mathrm{MHz}, \mathrm{CDCl}_{3}\right) ; 167.3,152.6,130.5,117.8,117.0,52.1$.

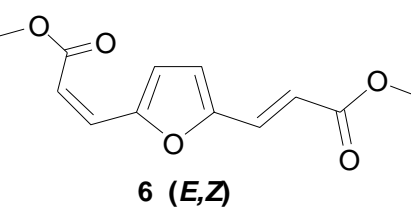

IR: 2923, 2853, 1727, 1635. MS: $m / z=\left[\mathrm{M}+\mathrm{NH}_{4}{ }^{+}\right] 254.2$ (100), 84.2 (88), 72.2 (98). HRMS: Calcd for $\mathrm{C}_{12} \mathrm{H}_{12} \mathrm{O}_{5}\left[\mathrm{M}+\mathrm{NH}_{4}^{+}\right]$: 254.1023. Found: 254.1024. $\delta_{\mathrm{H}}(400 \mathrm{MHz}$, $\left.\mathrm{CDCl}_{3}\right) ; 7.65(1 \mathrm{H}, \mathrm{d}, \mathrm{J} 3.7), 7.34(1 \mathrm{H}, \mathrm{d}, \mathrm{J} 15.7), 6.72(1 \mathrm{H}, \mathrm{d}, \mathrm{J} 12.9), 6.64(1 \mathrm{H}, \mathrm{d}, \mathrm{J}$ 3.7), $6.32(1 \mathrm{H}, \mathrm{d}, \mathrm{J} 15.7), 5.80(1 \mathrm{H}, \mathrm{d}, \mathrm{J} 12.9), 3.73(3 \mathrm{H}, \mathrm{s}), 3.71(3 \mathrm{H}, \mathrm{s}) . \delta_{\mathrm{C}}(400$ $\left.\mathrm{MHz}, \mathrm{CDCl}_{3}\right) ; 166.4,152.42,151.68,130.8,129.9,119.3,117.5,117.4,116.7,52.0$, 51.8. 


\section{3-[5-(3-Hydroxy-propyl)-furan-2-yl]-propan-1-ol}

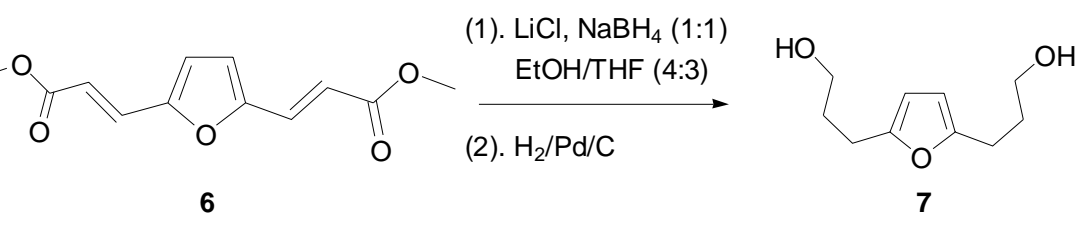

To a solution of $6(0.02 \mathrm{~g}, 0.085 \mathrm{mmol})$, in a (4:3) EtOH/THF solvent system (3mL) was added $\mathrm{LiCl}(0.072 \mathrm{~g}, 1.7 \mathrm{mmol}, 20 \mathrm{eq})$, and $\mathrm{NaBH}_{4}(0.064 \mathrm{~g}, 1.7 \mathrm{mmol}, 20 \mathrm{eq})$, and the reaction mixture was allowed to stir under argon at room temperature for $12 \mathrm{hrs}$. A saturated aqueous solution of sodium hydrogen carbonate $(2 \mathrm{~mL})$ was added. The aqueous layer was separated and extracted with $\mathrm{CH}_{2} \mathrm{Cl}_{2}(3 \times 10 \mathrm{ml})$. The combined organic extracts were washed with brine, dried over anhydrous $\mathrm{MgSO}_{4}$ and reduced in vacuo. Purification by column chromatography (eluting with 1:5 petrol ether/ethyl acetate) gave 3-[5-(3-Hydroxy-propyl)-furan-2-yl]-propan-1-ol 7 (0.011g, 71\%) as a colourless oil. IR: 3386.0, 2925.2, 1567.3, 1446.2, 1434.7, 1010.3, 785.3, 670.2. ). $\mathrm{m} / \mathrm{z}=[\mathrm{M}+\mathrm{H}] 202.2$ (27), 185.1 (100). HRMS: Calcd for $\mathrm{C}_{10} \mathrm{H}_{16} \mathrm{O}_{3}[\mathrm{M}+\mathrm{H}]$ : 185.1172. Found: 185.1172. $\delta_{\mathrm{H}}\left(400 \mathrm{MHz}, \mathrm{CDCl}_{3}\right) ; 5.88(2 \mathrm{H}, \mathrm{s}), 3.66(4 \mathrm{H}, \mathrm{t}, \mathrm{J}$ 6.4), $2.67(4 \mathrm{H}, \mathrm{t}, \mathrm{J} 7.4), 2.24(2 \mathrm{H}, \mathrm{s}), 1.87(4 \mathrm{H}, \mathrm{tt}, \mathrm{J} 6.4+7.4) . \delta_{\mathrm{C}}\left(400 \mathrm{MHz}, \mathrm{CDCl}_{3}\right) ; 153.9$, $105.4,61.9,30.9,24.3$.

\section{1,6,8-Trioxa-dispiro[4.1.4.2]tridec-12-ene}

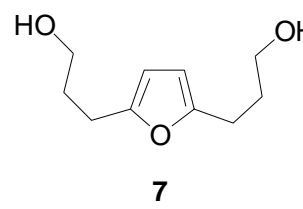

$\mathrm{OH}$

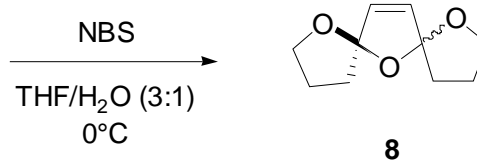

To a stirred solution of diol $7\left(0.035 \mathrm{~g}, 1.90 \times 10^{-4} \mathrm{M}\right)$ in a (3:1) $\mathrm{THF} / \mathrm{H}_{2} \mathrm{O}$ solvent system $(2 \mathrm{~mL})$ at $0^{\circ} \mathrm{C}$ was added portion wise over 20 minutes $N$-bromosuccinimide $(0.041 \mathrm{~g}$, $\left.2.3 \times 10^{-4} \mathrm{M}, 1.2 \mathrm{eq}\right)$. After the addition the reaction was allowed to stir for a further 20 minutes at $0^{\circ} \mathrm{C}$. A saturated aqueous solution of sodium thiosulphate $(1 \mathrm{~mL})$ was added and the mixture was allowed to stir for 5 minutes before the addition of a saturated aqueous solution of sodium hydrogen carbonate $(2 \mathrm{~mL})$ and diethyl ether $(5 \mathrm{~mL})$. The organic layer was separated, washed with a saturated aqueous solution of sodium hydrogen carbonate $(1 \times 5 \mathrm{~mL})$, dried over anhydrous sodium sulphate and reduced in vacuo. Purification by column chromatography over silica gel (eluting with 1:1 hexane/ethyl acetate) gave 1,6,8-trioxa-dispiro[4.1.4.2]tridec-12-ene 8 (0.016g, $46 \%$ ) as a colourless solid. IR: $2956,2853,1720,1456,1074.1021 . \mathrm{MS}: \mathrm{m} / \mathrm{z}=$ $[\mathrm{M}+\mathrm{H}]$ : 183.1 (100), 152.1 (32), 98.1 (32). HRMS: Calcd for $\mathrm{C}_{10} \mathrm{H}_{14} \mathrm{O}_{3}[\mathrm{M}+\mathrm{H}]$ : 
183.1016. Found: 183.1016. $\delta_{\mathrm{H}}\left(400 \mathrm{MHz}, \mathrm{C}_{6} \mathrm{D}_{6}\right) ; 5.81(1 \mathrm{H}, \mathrm{s}), 5.79(1 \mathrm{H}, \mathrm{s}), 4.02-$ $4.09(2 \mathrm{H}, \mathrm{m}), 3.68-3.74(2 \mathrm{H}, \mathrm{m}), 2.20-2.26(1 \mathrm{H}, \mathrm{m}), 1.95-2.07(2 \mathrm{H}, \mathrm{m}), 1.81-1.89(1 \mathrm{H}$, $\mathrm{m})$, 1.68-1.79 $(2 \mathrm{H}, \mathrm{m}), 1.51-1.62(2 \mathrm{H}, \mathrm{m}) ; \delta_{\mathrm{C}}\left(300 \mathrm{MHz}, \mathrm{C}_{6} \mathrm{D}_{6}\right) ; 132.11,131.82$, $116.28,115.28,66.98,66.92,35.93,35.62,23.69,23.64$.

\section{2,5-Bis-[4-(tert-butyl-dimethyl-silanyl-oxy)-but-1-enyl]-furan}

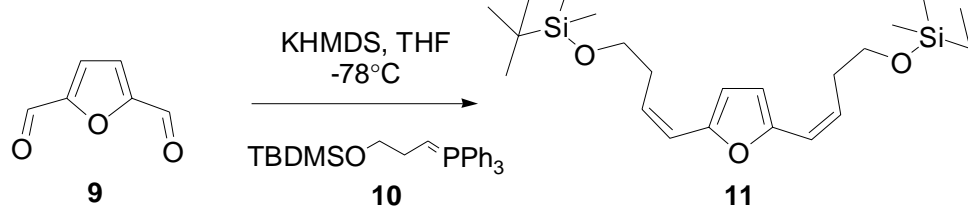

To a stirred suspension of phosphonium salt 10 (2.1g, 4 mmol, 2.2eq) in THF (30mL) at $-78^{\circ} \mathrm{C}$ under argon, was added a solution of KHMDS (0.5M solution in toluene, 8.1 $\mathrm{mL}, 4 \mathrm{mmol}, 2.2 \mathrm{eq}$ ) and the resulting orange mixture was stirred for 30 minutes. To this mixture was then added a solution of difuraldehyde $9(0.23 \mathrm{~g}, 1.8 \mathrm{mmol})$ in THF $(10 \mathrm{~mL})$ and the reaction was allowed to warm to room temperature over 2 hours. A saturated aqueous solution of ammonium chloride $(20 \mathrm{~mL})$ was added, followed by ethyl acetate $(20 \mathrm{~mL})$. The aqueous layer was separated and extracted with ethyl acetate $(3 \times 20 \mathrm{~mL})$ and the combined organic extracts were washed with a saturated aqueous solution of $\mathrm{NaHCO}_{3}$ and brine, dried over anhydrous $\mathrm{MgSO}_{4}$ and reduced in vacuo. Purification by column chromatography (2 columns firstly eluting with 1:1 petrol ether/ethyl acetate, then 10:1 petrol ether/ethyl acetate) gave 2,5-Bis-[4-(tertbutyl-dimethyl-silanyl-oxy)-but-1-enyl]-furan $11(0.42 \mathrm{~g}, 54 \%)$ as a yellow oil. IR: 2954.8, 2927.8, 2856.2, 1700.1, 1652.6. MS: $m / z=[M+H]: ~ 458.4$ (40), 454.4 (70), 69.3 (100). HRMS: Calcd for $\mathrm{C}_{24} \mathrm{H}_{44} \mathrm{O}_{3} \mathrm{Si}_{2}\left[\mathrm{M}+\mathrm{NH}_{4}{ }^{+}\right]$: 454.3167. Found: 454.3168. $\delta_{\mathrm{H}}$ (400 MHz, $\left.\mathrm{CDCl}_{3}\right) ; 6.24(2 \mathrm{H}, \mathrm{s}), 6.19(2 \mathrm{H}, \mathrm{dt}, \mathrm{J} 9.80,1.46), 5.57(2 \mathrm{H}, \mathrm{dt}, \mathrm{J} 9.8,6.0)$, $3.72(4 \mathrm{H}, \mathrm{t}, J 5.5), 2.69(4 \mathrm{H}, \mathrm{dt}, 5.5,1.4), 0.87(18 \mathrm{H}, \mathrm{s}), 0.04(12 \mathrm{H}, \mathrm{s}) . \delta_{\mathrm{C}}(400 \mathrm{MHz}$, $\left.\mathrm{CDCl}_{3}\right) ; 152.18,127.10,118.41,110.97,62.65,32.98,25.93,18.33,-5.28$. 
2,5-Bis-[4-(tert-butyl-dimethyl-silanyloxy)-butyl]-furan

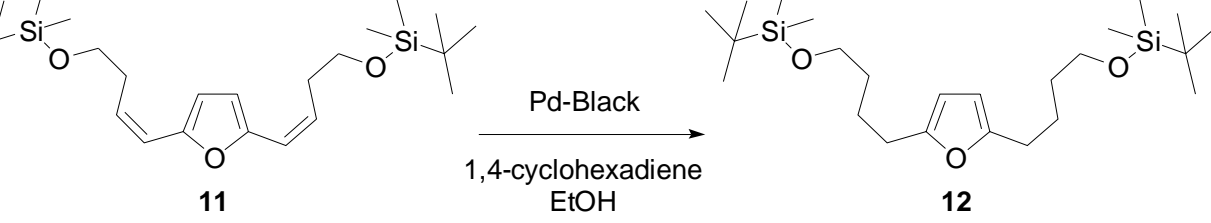

A solution of di-olefin $(0.15 \mathrm{~g}, 0.34 \mathrm{mmol})$ in $\mathrm{EtOH}(20 \mathrm{~mL})$, under argon at room temperature was treated with Pd-Black (0.6g, 4 eq by weight) and 1,4cyclohexadiene $(1.1 \mathrm{~g}, 13.7 \mathrm{mmol}, 40 \mathrm{eq})$ and the reaction was stirred at room temperature for 2 hours. The reaction mixture was filtered through celite and reduced in vacuo to give 2,5-Bis-[4-(tert-butyl-dimethyl-silanyloxy)-butyl]-furan 12 (0.148g, 98\%) as a colourless oil. IR: 2927.8, 2856.8, 1471.5, 1462.7, 1388.5, 1360.8, 1256.2, 1103.9. MS: $m / z=\left[M+N_{4}\right]: 458.4$ (100), 69.3 (41), 63.3 (46). HRMS: Calcd for $\mathrm{C}_{24} \mathrm{H}_{48} \mathrm{O}_{3} \mathrm{Si}_{2}\left[\mathrm{M}+\mathrm{NH}_{4}{ }^{+}\right]:$458.3480. Found: 458.3486. $\delta_{\mathrm{H}}\left(400 \mathrm{MHz}, \mathrm{CDCl}_{3}\right) ; 5.84(2 \mathrm{H}$, s), $3.63(4 \mathrm{H}, \mathrm{t}, \mathrm{J} 6.3), 2.58(4 \mathrm{H}, \mathrm{t}, \mathrm{J} 7.4), 1.62-1.70(4 \mathrm{H}, \mathrm{m}), 1.53-1.60(4 \mathrm{H}, \mathrm{m}), 0.9$ $(18 \mathrm{H}, \mathrm{s}), 0.04(12 \mathrm{H}, \mathrm{s}) . \delta_{\mathrm{C}}\left(400 \mathrm{MHz}, \mathrm{CDCl}_{3}\right) ; 154.37,104.97,62.91,32.31,27.80$, $25.96,24.42,18.35,-5.30$.

\section{4-[5-(4-Hydroxy-butyl)-furan-2-yl]-butan-1-ol}

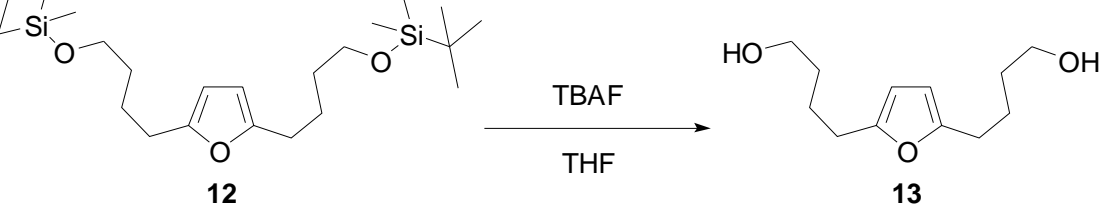

A solution of silyl ether $12(0.135 \mathrm{~g}, 0.31 \mathrm{mmol})$ in THF $(5 \mathrm{~mL})$ under an atmosphere of argon at room temperature was treated with a solution of tetra- ${ }^{n}$ butyl ammonium fluoride (1M in THF, $0.67 \mathrm{~mL}, 2.2 \mathrm{eq}$ ) and the reaction mixture was allowed to stir at room temperature for 2 hours before the addition of brine $(2 \mathrm{~mL})$. The aqueous layer was separated and extracted with diethyl ether $(3 \times 10 \mathrm{~mL})$. The combined extracts were washed with saturated $\mathrm{NaHCO}_{3}$, dried over anhydrous $\mathrm{MgSO}_{4}$ and reduced in vacuo. Purification by column chromatography (eluting with 1:5 petrol ether/ethyl acetate) gave 4-[5-(4-Hydroxy-butyl)-furan-2-yl]-butan-1-ol $13(0.035 \mathrm{~g}, 54 \%)$ as a colourless oil. IR: $3337.3,2925.6,1652.6,1559.7,1456.5,1063.1 . M S: ~ m / z=[M+H]$ : 213.1 (100), 195.1 (20). HRMS: Calcd for $\mathrm{C}_{12} \mathrm{H}_{20} \mathrm{O}_{3}\left[\mathrm{M}+\mathrm{H}^{+}\right]$: 213.1485. Found: 213.1483. $\delta_{\mathrm{H}}\left(400 \mathrm{MHz}, \mathrm{CDCl}_{3}\right) ; 5.84(2 \mathrm{H}, \mathrm{s}), 3.63(4 \mathrm{H}, \mathrm{t}, \mathrm{J} 6.3), 2.59(4 \mathrm{H}, \mathrm{t}, \mathrm{J} 7.2)$, 1.64-1.71 (4H, m), 1.55-1.62 (4H, m). $\delta_{\mathrm{C}}\left(400 \mathrm{MHz}, \mathrm{CDCl}_{3}\right) ; 154.14,105.26,62.64$, $32.12,27.70,24.35$. 


\section{1,7,9-Trioxa-dispiro[5.1.5.2]pentadec-14-ene}

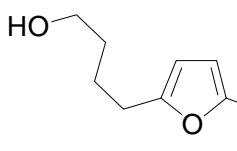

13

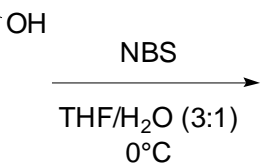

$0^{\circ} \mathrm{C}$

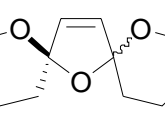

14

To a stirred solution of diol $13\left(0.045 \mathrm{~g}, 2.12 \times 10^{-4} \mathrm{M}\right)$ in a $(3: 1) \mathrm{THF} / \mathrm{H}_{2} \mathrm{O}$ solvent system $(2 \mathrm{~mL})$ at $0^{\circ} \mathrm{C}$ was added portion wise over 20 minutes $N$-bromosuccinimide $\left(0.045 \mathrm{~g}, 2.5 \times 10^{-4} \mathrm{M}, 1.2 \mathrm{eq}\right)$. After addition the reaction was allowed to stir for a further 20 minutes at $0^{\circ} \mathrm{C}$. A saturated aqueous solution of sodium thiosulphate $(1 \mathrm{~mL})$ was added and the mixture was allowed to stir for 5 minutes before the addition of a saturated aqueous solution of sodium hydrogen carbonate $(2 \mathrm{~mL})$ and diethyl ether $(5 \mathrm{~mL})$. The organic layer was separated, washed with a saturated aqueous solution of sodium hydrogen carbonate $(1 \times 5 \mathrm{~mL})$, dried over anhydrous sodium sulphate and reduced in vacuo. Purification by column chromatography over silica gel (eluting with 1:1 hexane/ethyl acetate) gave 1,7,9-Trioxa-dispiro[5.1.5.2]pentadec-14-ene 14 $(0.029 \mathrm{~g}, 65 \%)$ as a colourless solid. IR: 2940, 2870, 1653. MS: $m / z=[\mathrm{M}+\mathrm{H}] 161.0$ (100), 211.1 (80). HRMS: Calcd for $\mathrm{C}_{12} \mathrm{H}_{18} \mathrm{O}_{3}[\mathrm{M}+\mathrm{H}]$ : 211.1329. Found: 211.1329. $\delta_{\mathrm{H}}$ (400 MHz, $\left.\mathrm{C}_{6} \mathrm{D}_{6}\right) ; 5.93(1 \mathrm{H}, \mathrm{s}), 5.90(1 \mathrm{H}, \mathrm{s}), 3.98-4.06(2 \mathrm{H}, \mathrm{m}), 3.70-3.82(2 \mathrm{H}, \mathrm{m})$, 1.86-1.98 $(2 \mathrm{H}, \mathrm{m}), 1.51-1.79(10 \mathrm{H}, \mathrm{m}) ; \delta_{\mathrm{C}}\left(400 \mathrm{MHz}, \mathrm{C}_{6} \mathrm{D}_{6}\right) ; 134.05,133.99,109.71$, $106.85,63.66,63.50,35.30,34.76,25.21,25.15,19.84,19.77$. 


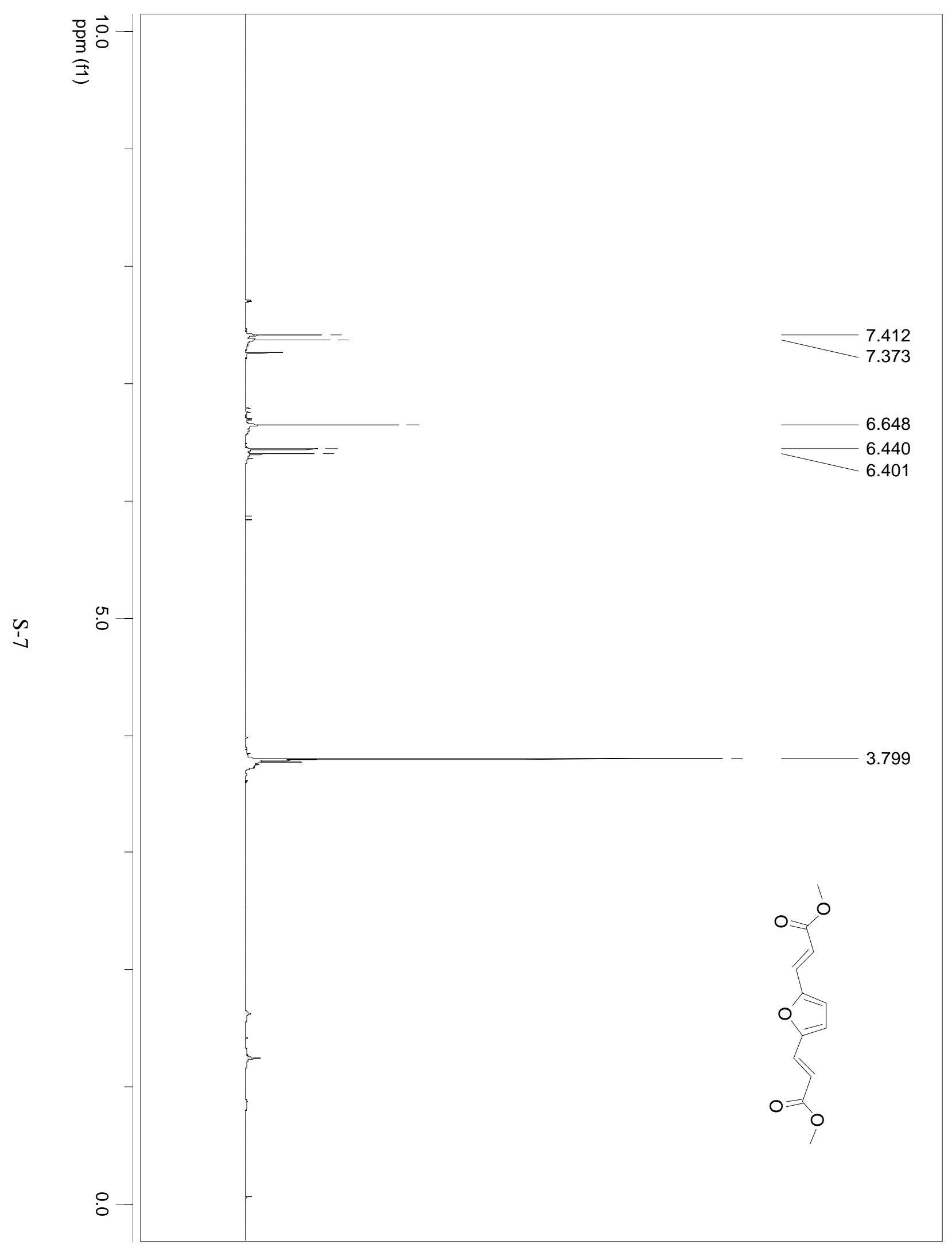

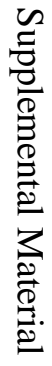




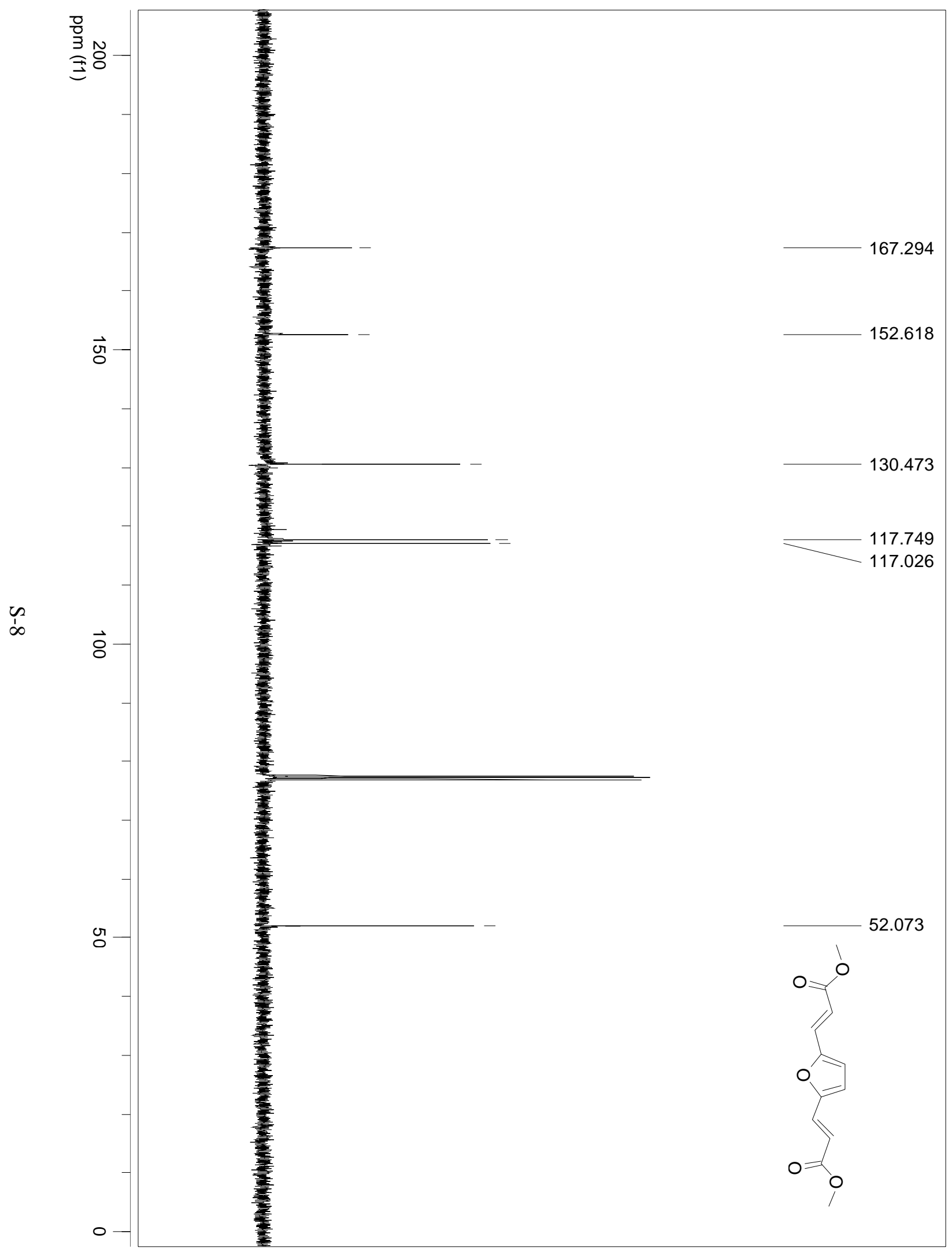

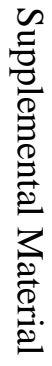




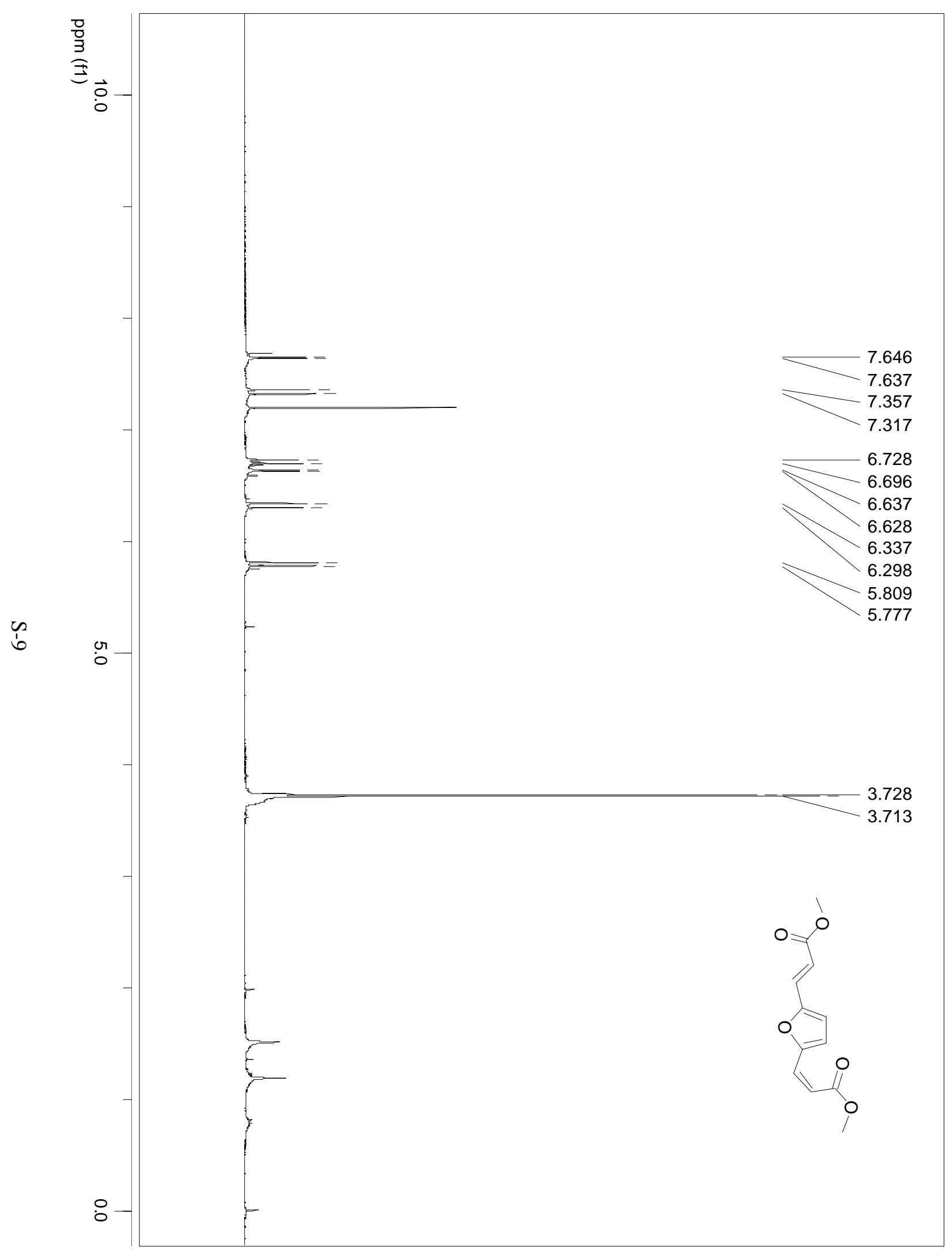




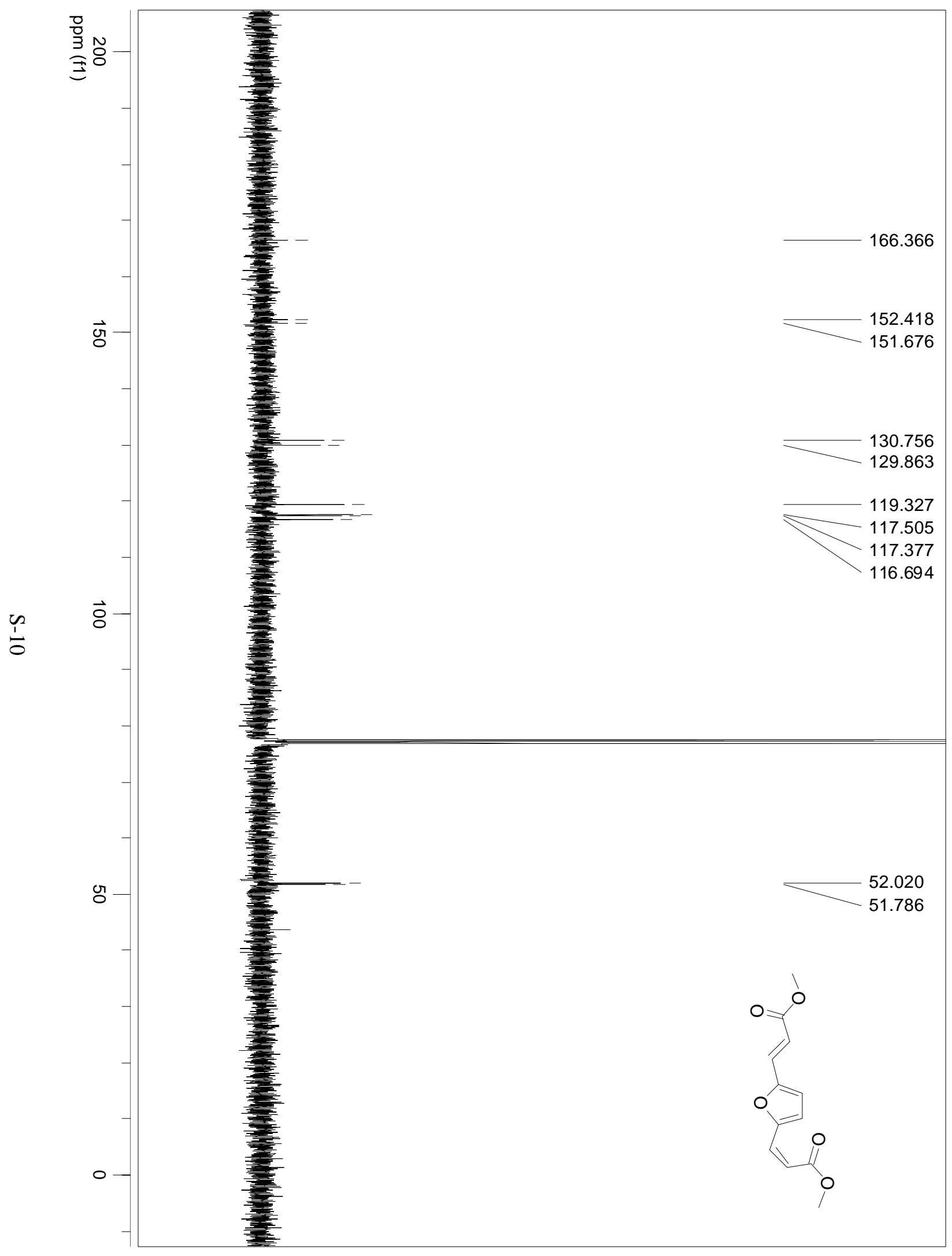




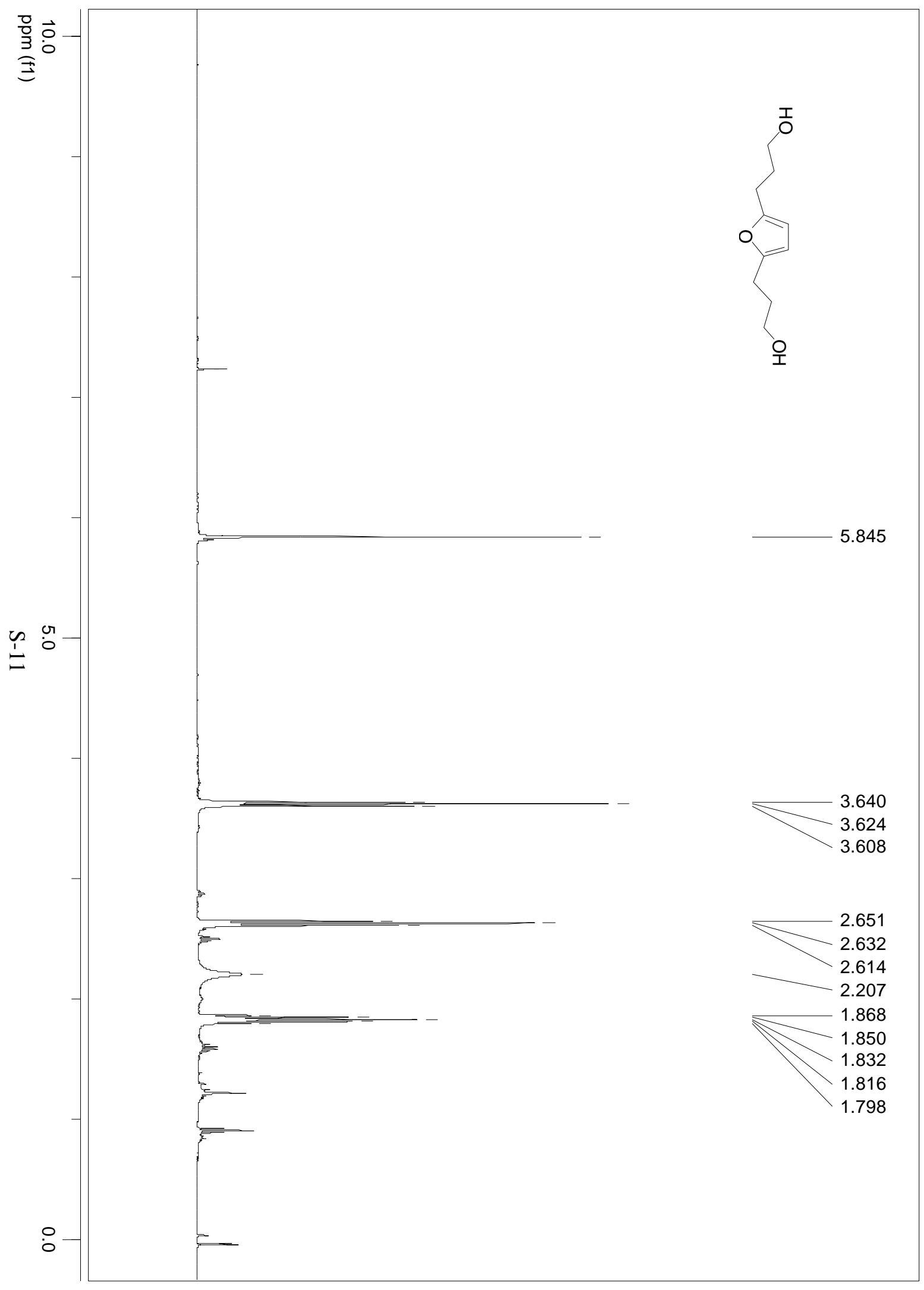

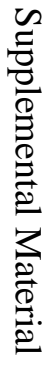




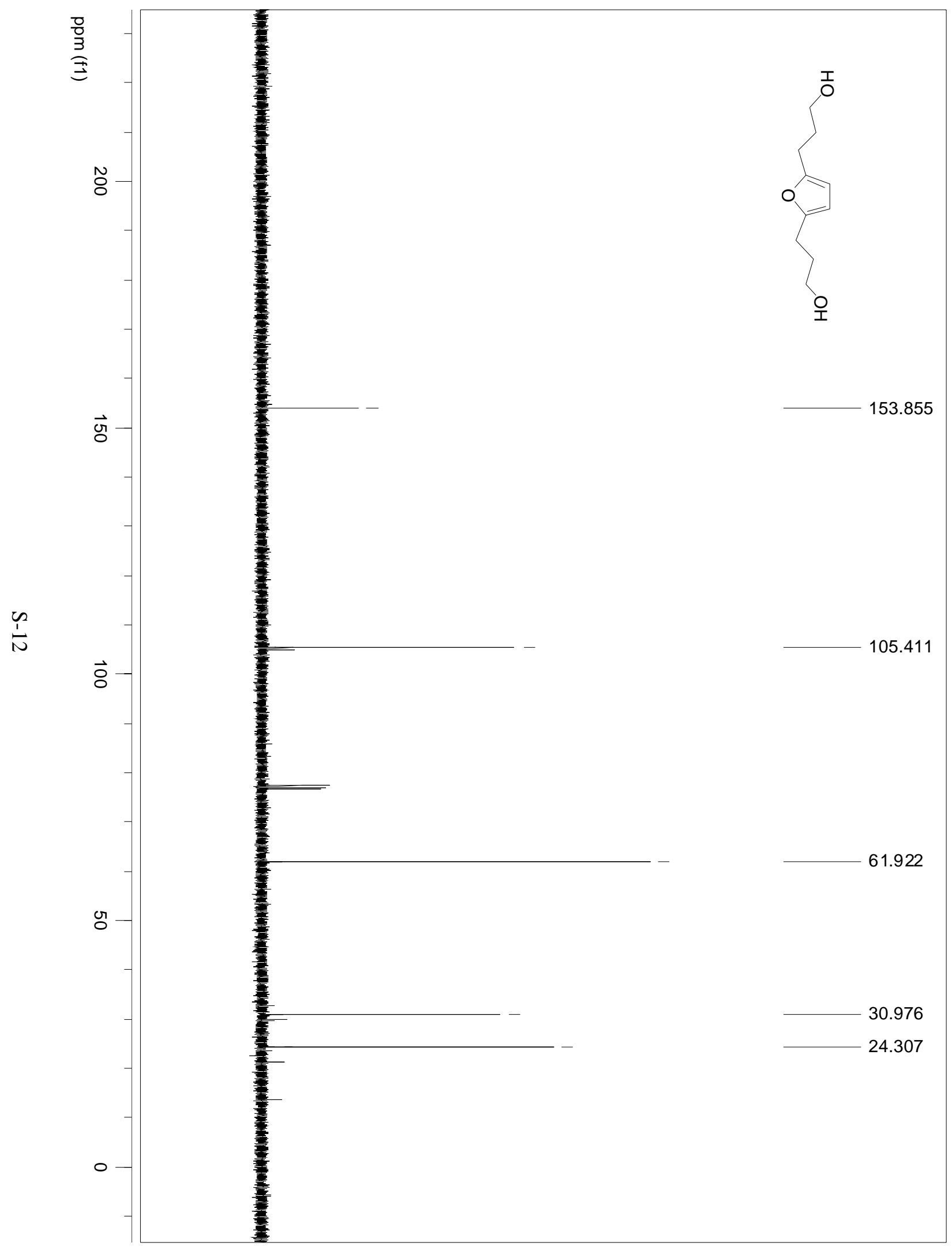




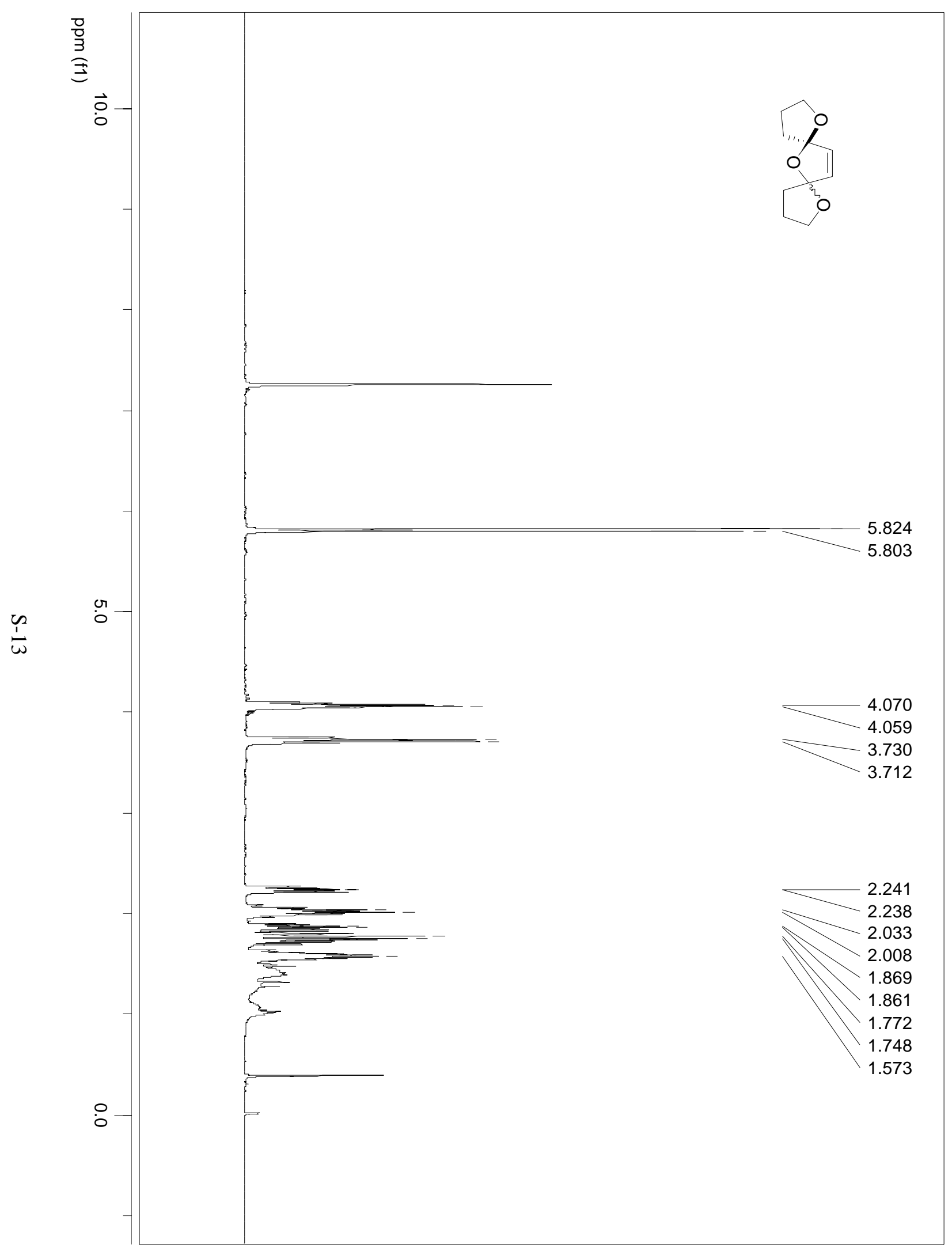




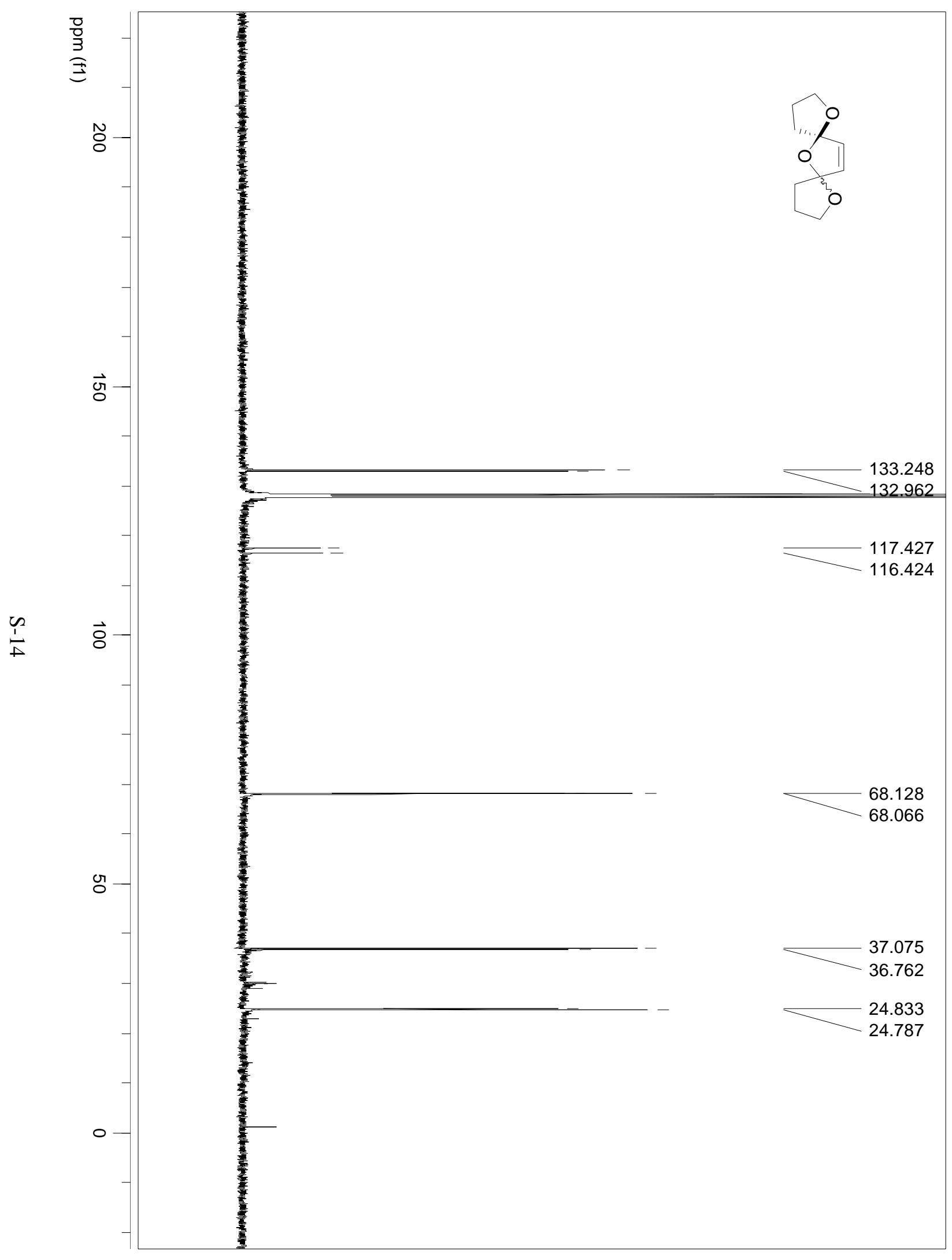

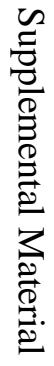




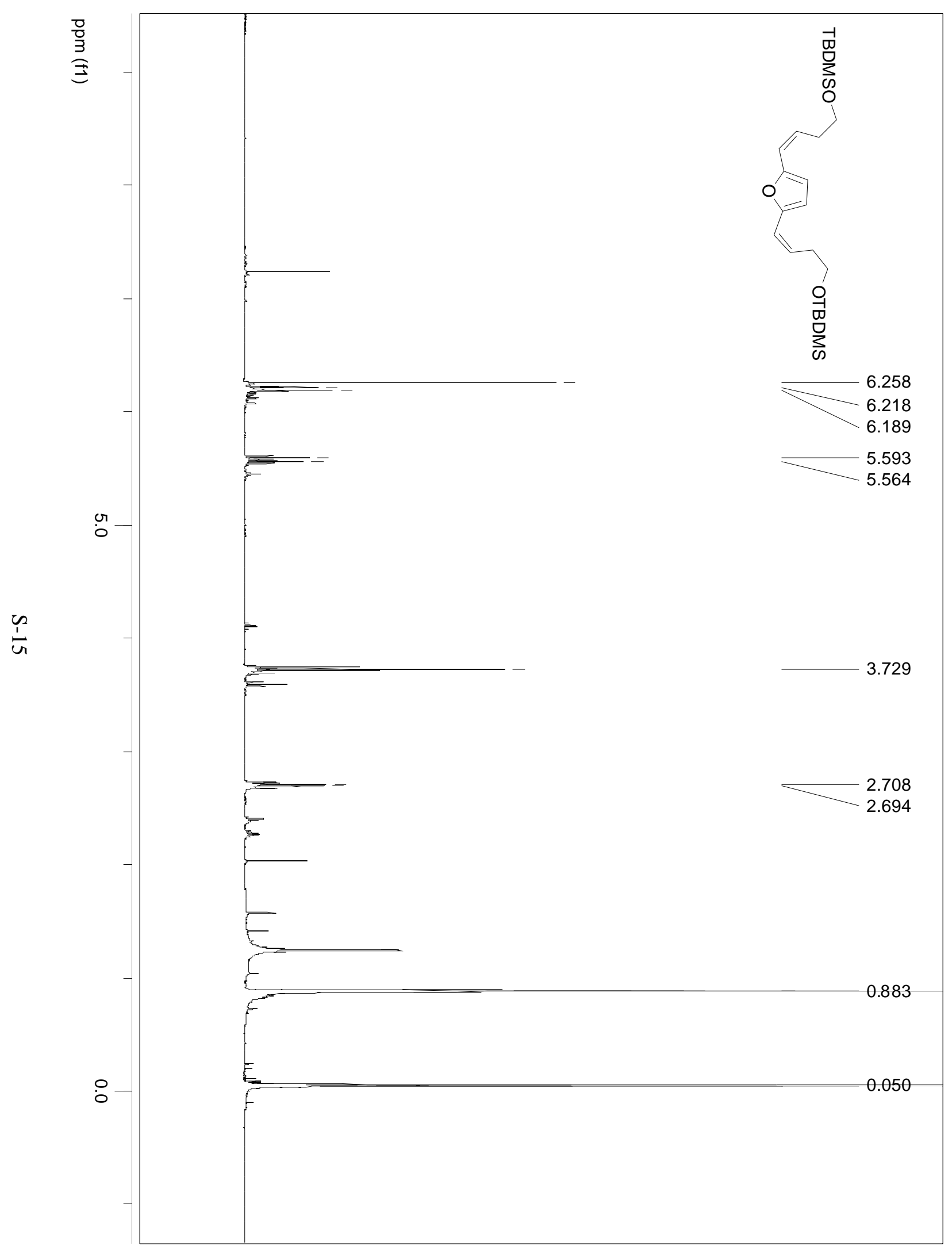

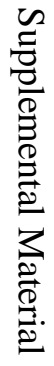




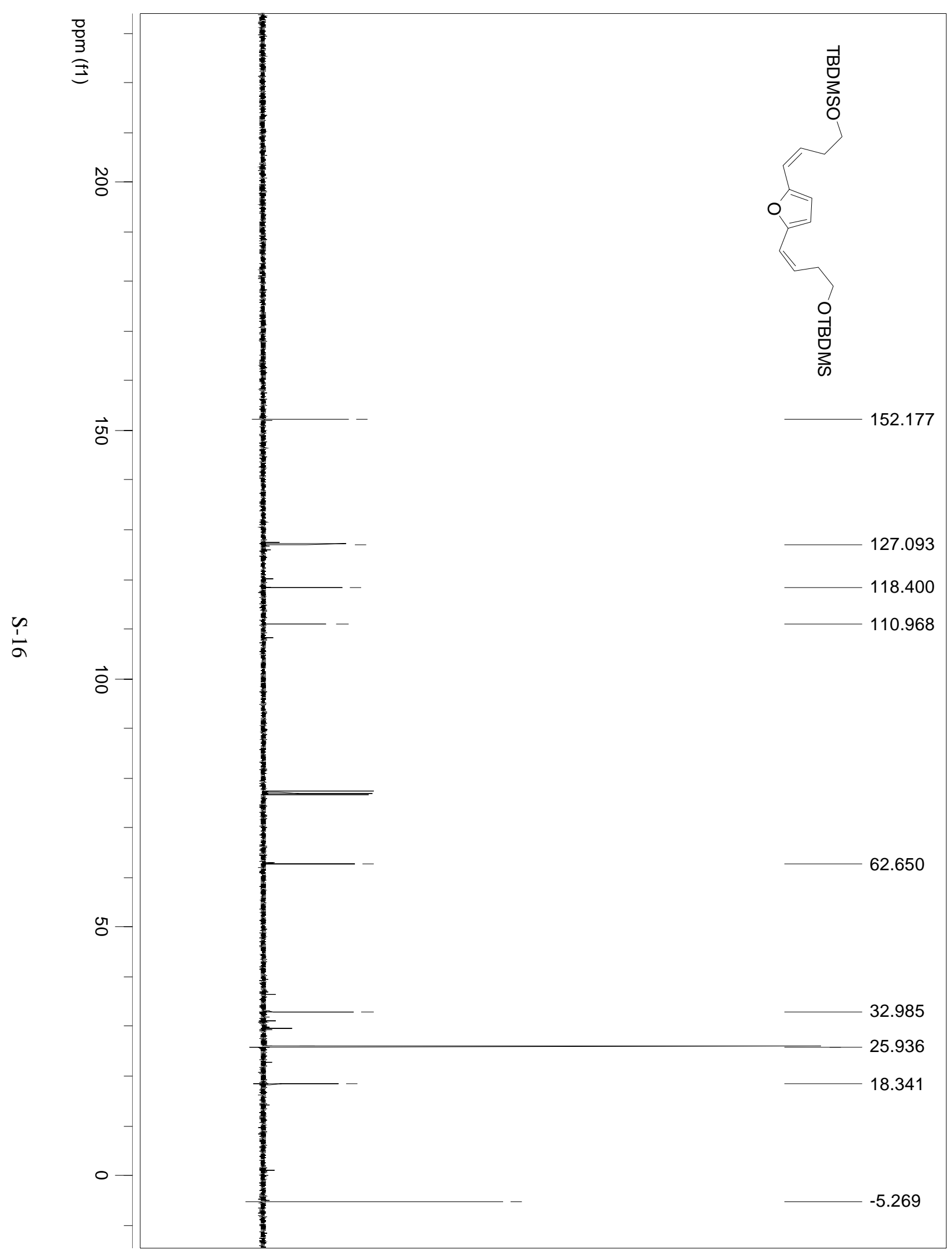




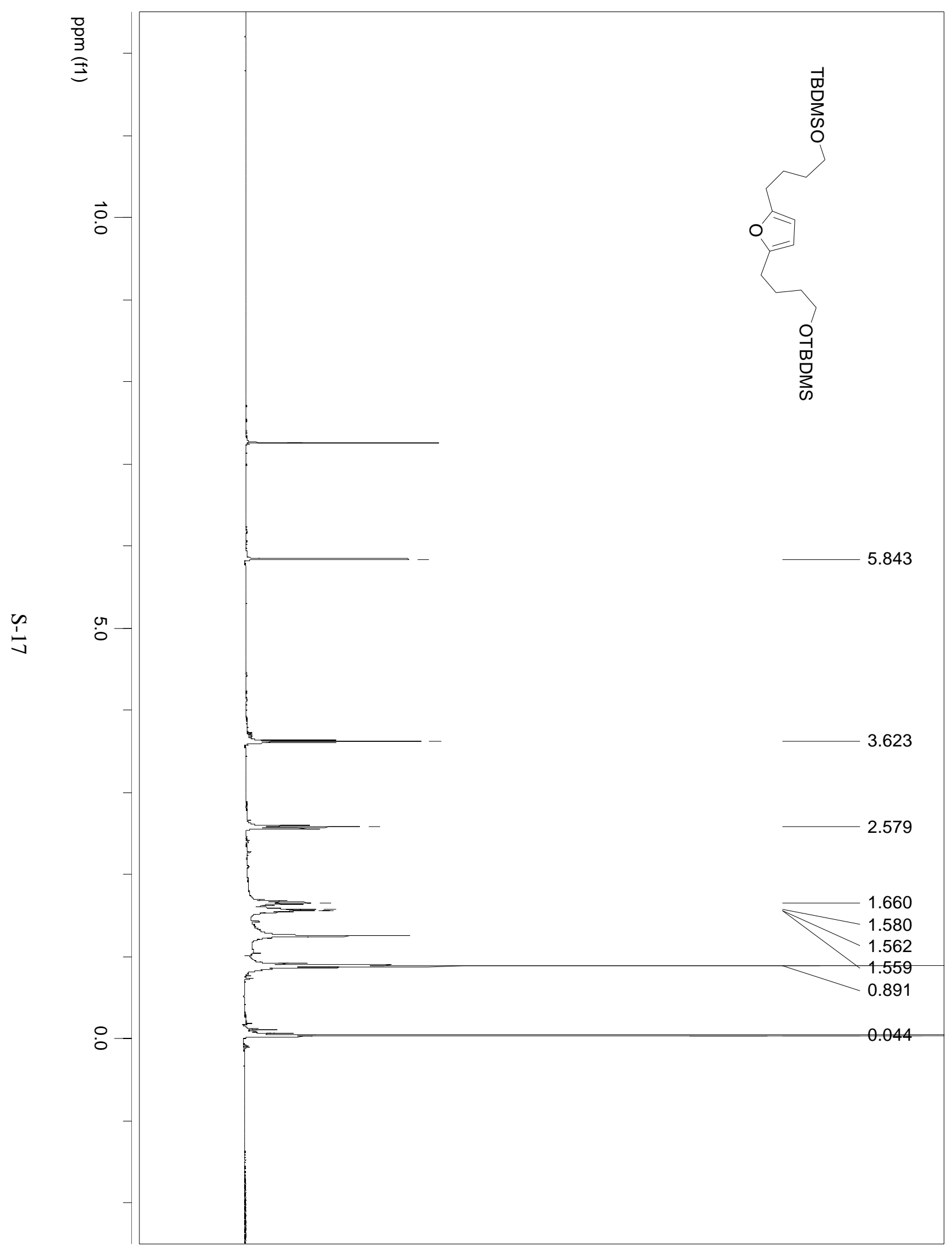




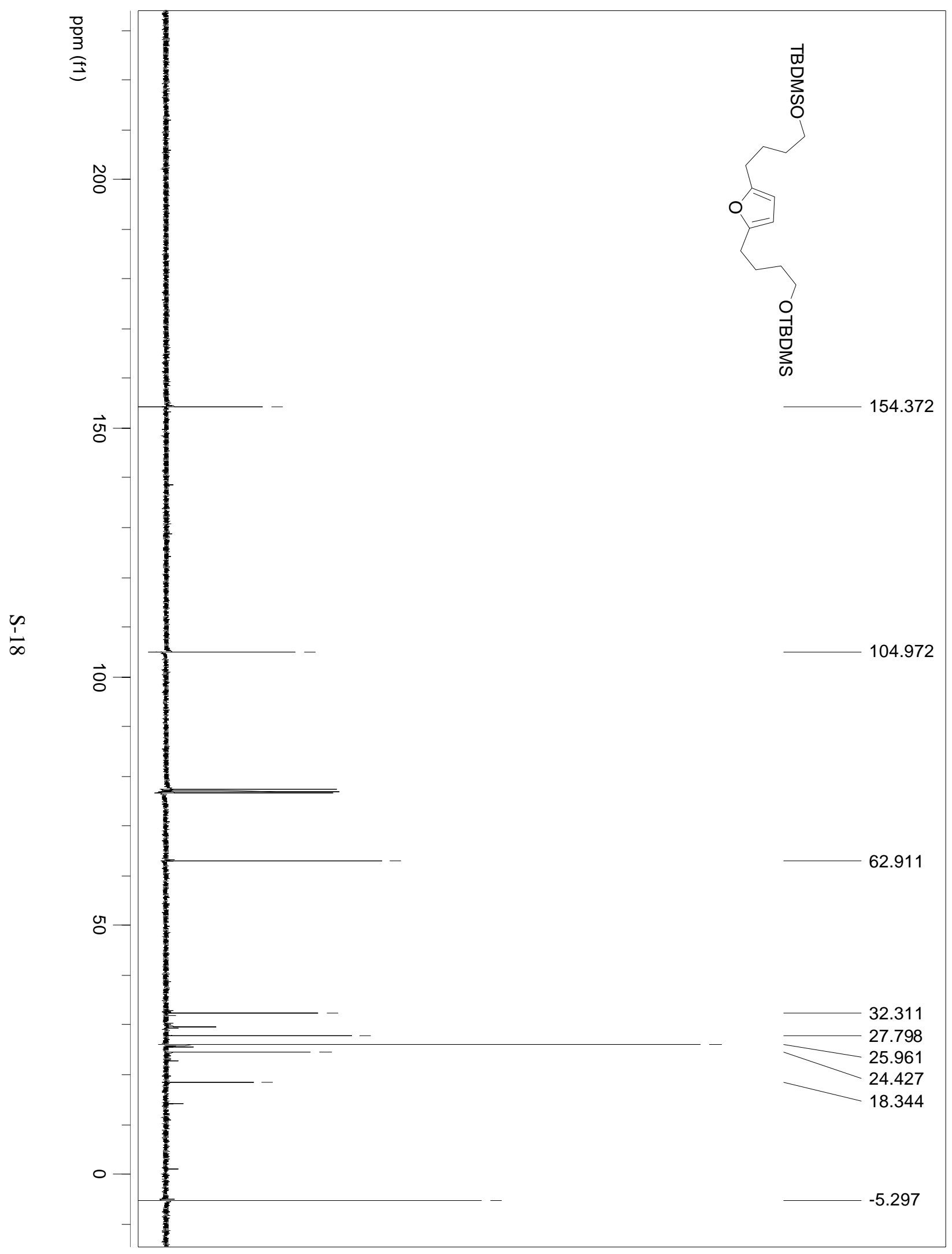




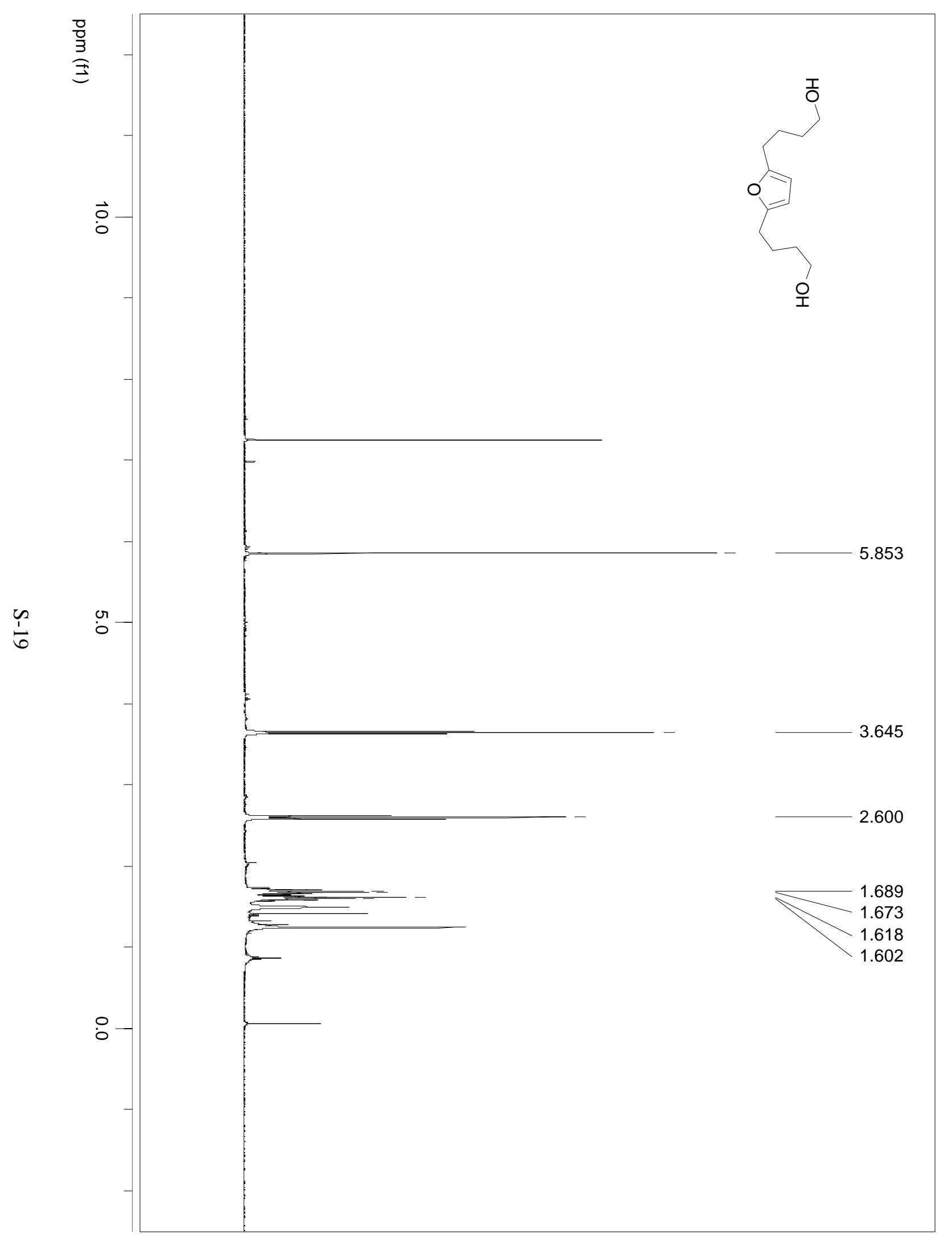




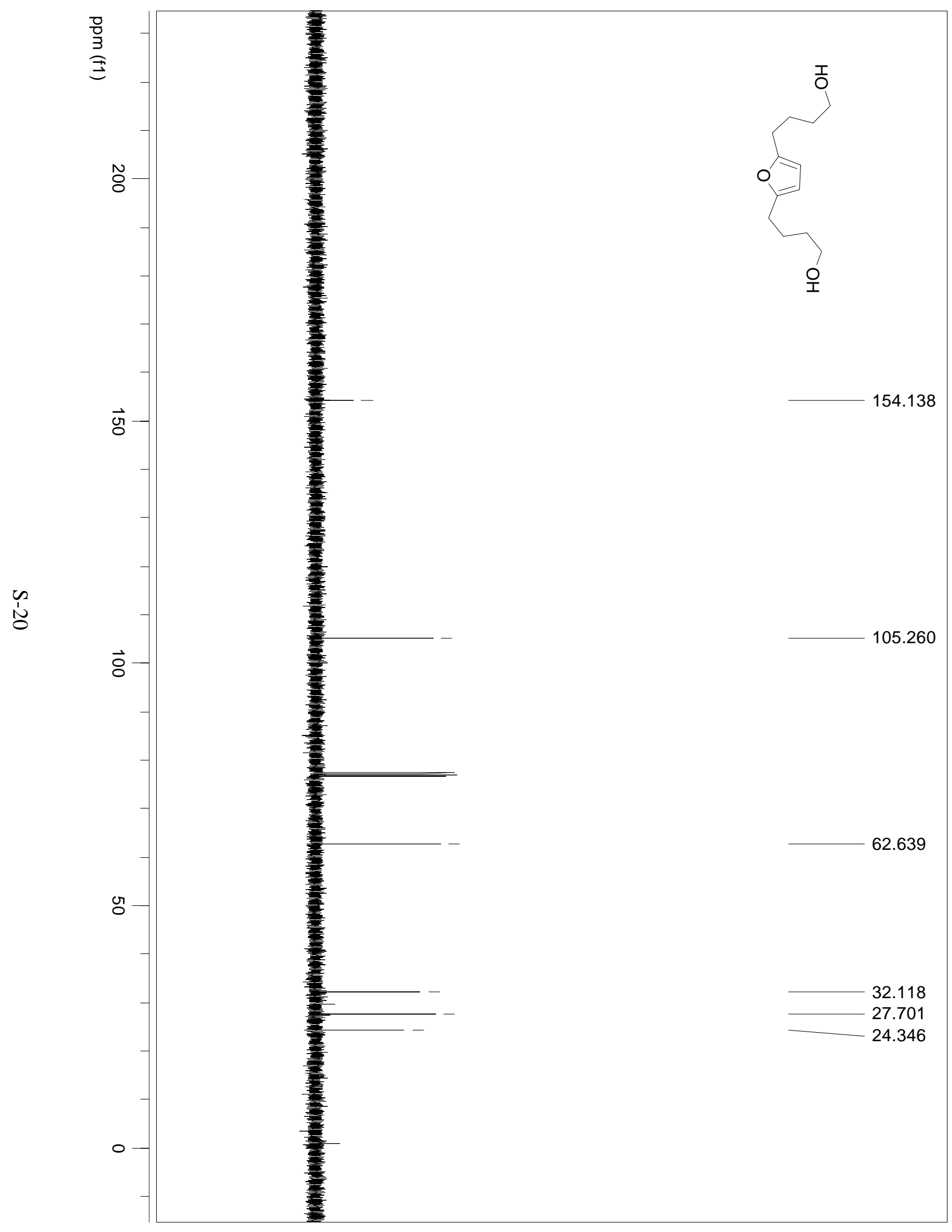




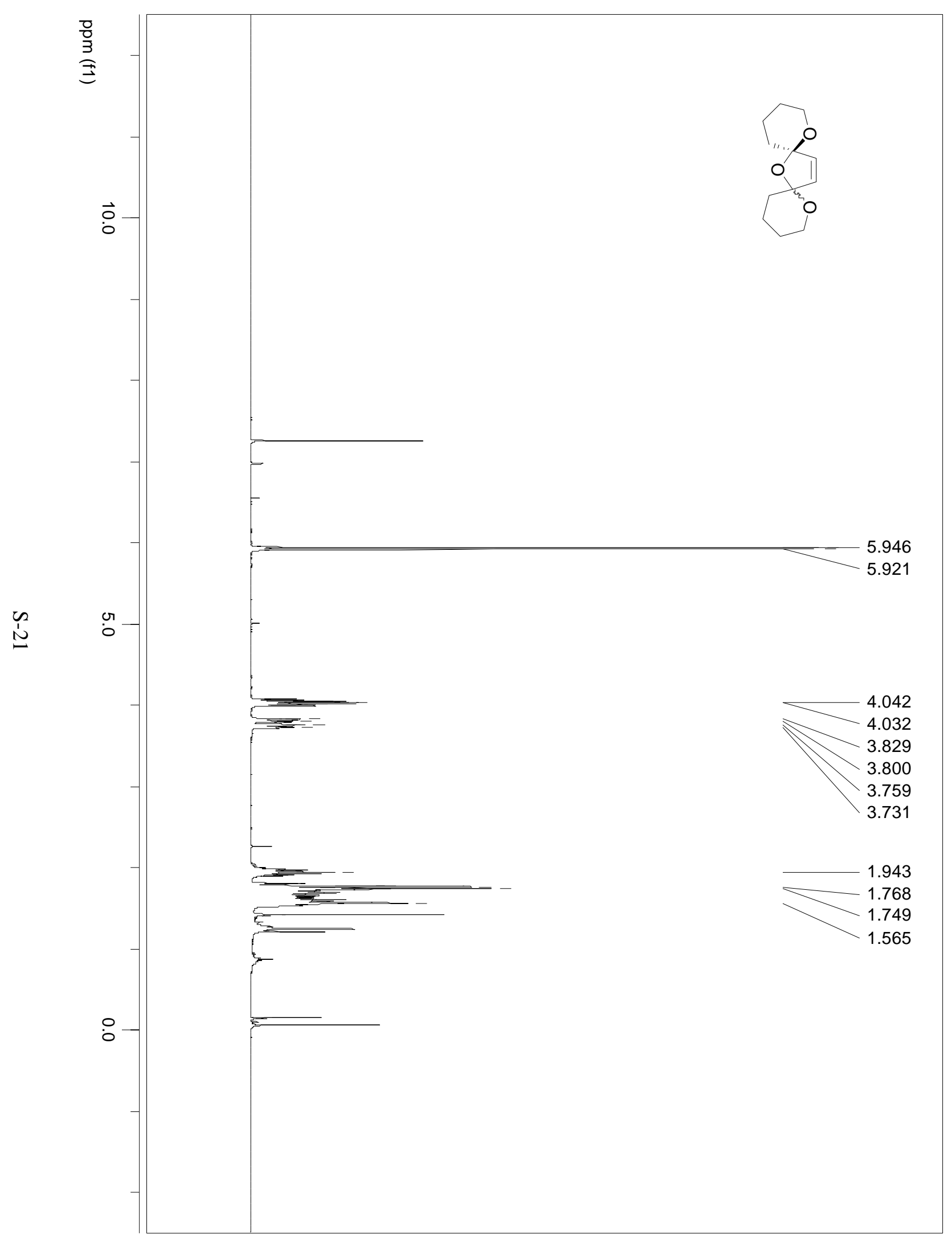

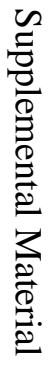




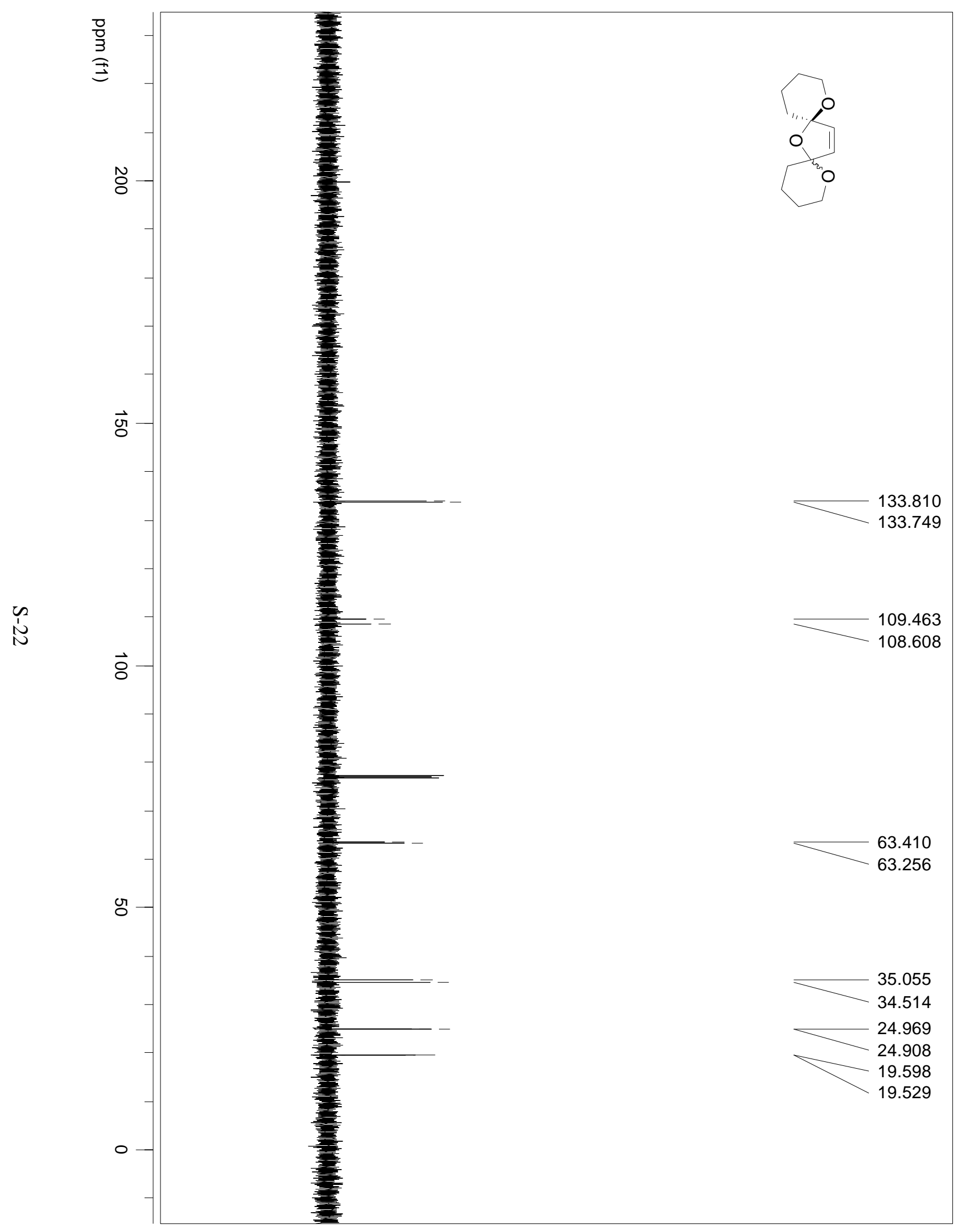

